


\title{
EL AGRONEGOCIO SOJERO EN ARGENTINA: MODELO EXTRACTIVO EN LOS MUNDOS RURALES
}

\author{
TOMÁS PALMISANO \\ Universidad de Buenos Aires, Argentina
}

Recepción manuscrito: 15 de marzo de 2016

Aceptación versión final: 29 de abril de 2016

\begin{abstract}
RESUMEN La extensión del neoliberalismo en Argentina tuvo múltiples aristas. En el caso de la producción agropecuaria se tradujo en la extensión de un modelo productivo que reconfiguró los mundos rurales. La hipótesis de este trabajo es que el agronegocio, concepto con que se conoce a este modelo, puede entenderse como una manifestación específica del extractivismo. A partir de estadísticas públicas y trabajos académicos proponemos una reconstrucción de este proceso a fin de justificar el carácter extractivo del agronegocio y así conectarlo a las dinámicas más amplias que marcan la economía global contemporánea.
\end{abstract}

PALABRAS ClAVE Argentina, mundo rural, agronegocio, extractivismo, neoliberalismo, economía global.

\begin{abstract}
The spreading of neoliberalism in Argentina had a number of edges. Regarding agriculture and livestock production, it became a productive model that put together the rural worlds once more. The hypothesis of this work is that agribusiness (farming business), the term by which this model is known, can be understood as a specific manifestation of extractivism (excessive extraction). Based on public statistics and academic papers, we propose a reconstruction of this process in order to justify the extractive nature of agribusiness, and, in this way, connect it to the most ample dynamics that determine contemporary global economy.

KEYWORDS Argentina, rural world, agribusiness, extractivism, neoliberalism, global economy.

Jel CODES E65, N56, Q33.
\end{abstract}

\section{INTRODUCCIÓN}

En la década de 1970 la economía mundial sufrió una intensificación del proceso de internacionalización de las transacciones comerciales y financieras que requirió un nuevo «aporte» de recursos de los países «en vías de desarrollo». Este proceso se imbricó con el paradigma neoliberal, bajo el cual se extendió la concentración del capital en grandes empresas que dominaban múltiples escalas de producción y que en muchos casos se centraban en los procesos primario-exportadores. Lo particular de estos últimos años fue el recrudecimiento de una lógica capitalista que buscaba mercantilizar territorios, tecnologías y estrategias de 
producción locales y de poblaciones culturalmente ricas y diversas en tierra, capital y trabajo. En el caso de la producción minera, los cambios tecnológicos de la modalidad a cielo abierto con utilización de sustancias tóxicas reconfiguraron el mapa económico-político de cada lugar donde la concentración de minerales hizo redituable la explotación. Reflotando el mito de los desiertos, los territorios potencialmente explotables se presentan como vacíos o sacrificables (Svampa, Bottaro y Sola Álvarez, 2009), ocultando las redes sociales, identitarias y productivas que allí se imbrican. Para el caso de la agricultura, la expansión de los cultivos para la exportación implicó la subordinación mercantil de espacios comunes tanto naturales - bosques nativos, selvas, cerrados, mesetas semidesérticas, valles andinos, etc.-, como productivos destinados al autoconsumo. La extensión en el campo argentino del agronegocio, concepto con el que Giarracca y Teubal (2008) identifican este modelo, tuvo una profunda complejidad.

En este contexto, la hipótesis del artículo es que el agronegocio en su versión sojera es una manifestación específica de una dinámica económica global más amplia de extensión del modelo extractivo. Para contrastar esta conjetura proponemos desarrollar algunas dimensiones del modelo extractivo a partir de elementos empíricos presentes en las transformaciones agrarias argentinas de los últimos tiempos. En términos metodológicos se recurrirá principalmente a datos de tipo cuantitativo ya sea procesados a partir de bases de datos de acceso público como a los que se encuentran disponibles en la extensa bibliografía sobre el tema.

\section{MARCO TEÓRICO}

El concepto de acumulación por desposesión de Harvey (2004) se inserta en una vertiente del pensamiento crítico $^{1}$ que aborda la convivencia de la acumulación ampliada del capital y los procesos de desposesión típicos de la acumulación originaria (Marx, 2006). Según el autor, desde la década de 1970 el capitalismo global está inmerso en una crisis de sobreacumulación que pudo ser resuelta por medio de un modelo de acumulación ampliada sostenible y, por tanto, se han intensificado los procesos de despojo.

En esta nueva etapa, a las prácticas de expoliación más tradicionales — privatización de la tierra, expulsión de poblaciones, mercantilización del trabajo, guerras, deudas públicas, difusión del capital financiero- se sumaron otras novedosas: el énfasis en los derechos de propiedad intelectual, patentes y licencias de materiales genéticos, plasma de semillas y diversas formas de biodiversidad conservadas por poblaciones locales; la depredación de los bienes ambientales globales — tierra, aire, agua - y la degradación ambiental; la mercantilización de las formas culturales, las historias y la creatividad intelectual de los pueblos; la corporativización y privatización de activos previamente públicos — universidades, servicios públicos, empresas del Estado- (Harvey, 2004, p. 114). El planteamiento de Harvey supone una lectura de la actualidad del capitalismo en su nivel global por lo cual consideramos indispensable combinarlo con elementos adicionales, muchos de ellos desarrollados desde una perspectiva latinoamericana que nos permiten insertar la categoría de extractivismo o modelo extractivo.

En primer lugar, puede destacarse un aumento del precio de los commodities durante la primera década del siglo xxi. Como plantean Giarracca y Teubal (2013), Headey y Fan (2010) y Kosacoff y Campanario (2007), los principales componentes de la canasta alimentaria y la 
gran mayoría de los metales, mostraron valores absolutos altos que empujaron la frontera productiva y la expansión de las actividades extractivas. La extensión de estas actividades consolidaron lo que Svampa (2012) denomina «consenso de los commodities», entendido como «el ingreso a un nuevo orden económico y político, sostenido por el boom de los precios internacionales de las materias primas y los bienes de consumo, demandados cada vez más por los países centrales y las potencias emergentes» (p. 16). La reprimarización de las economías y la profundización de una dinámica de desposesión muestran, para la autora, un estilo de desarrollo extractivista asentado en la sobreexplotación de los recursos naturales y la expansión de la frontera productiva. En este marco, la idea de «consenso de los commodities» combina estas características productivas con una narrativa ambivalente y contradictoria que entrelaza la ideología neoliberal y el neodesarrollismo progresista.

En segundo lugar, la dislocación traumática y las rebeliones contra las expresiones más crudas del neoliberalismo - el levantamiento zapatista de 1994 en México, las manifestaciones antiglobalización de 1999 en Seattle, la rebelión argentina de 2001 y 2002, son algunos ejemplos de ello- obligaron a los actores económicos hegemónicos a transmutar sus esquemas explicativos y adaptarse a coyunturas políticas diversas haciendo hincapié en conceptos como la participación, el empoderamiento y el desarrollo local (Montenegro, 2011, 2008; Tussie, 2000). Murillo (2008) plantea que incluso el Banco Mundial (BM) incorporó

el concepto sumamente sugerente de «aprendizaje», el cual sostiene de manera explícita el abandono de cualquier modo rígido en las estrategias discursivas. Como veremos, «escuchar la voz de los pobres», dar un lugar preferencial al triálogo con la sociedad civil se han tornado tópicos centrales de su estrategia discursiva. (p. 43)

En paralelo, las empresas intensificaron sus estrategias consensuales. Así, los continuos llamados a la responsabilidad social empresarial mostraron la necesidad de dotar de un rostro más amigable a los emprendimientos, desde los más «políticamente correctos» a los más depredatorios. Con ello se pone en manos de la voluntad de las corporaciones la mejora en las condiciones sociales, económicas y ambientales de los territorios donde operan e incluso fuera de ellos (Banerjee, 2010; Cafiero, 2010).

Por otro lado, el concepto de neoextractivismo progresista de Gudynas (2009) pone en relieve el contexto sociopolítico de América Latina, donde ascendieron numerosos gobiernos que desde las prácticas y/o sus discursos buscaron diferenciarse de las anteriores políticas neoliberales. Bolivia, Venezuela y Ecuador fueron los ejemplos más críticos, mientras que Brasil, Argentina, Uruguay y Perú - junto al período de gobierno de Michelle Bachelet en Chile y Fernando Lugo en Paraguay - tuvieron reformas más matizadas. Salvando las profundas diferencias, estas administraciones han sido muy críticas a la primacía del mercado como asignador privilegiado de recursos y han intentado avanzar hacia políticas de ampliación de derechos económicos, políticos, culturales y sociales, dándole al Estado un rol primordial. Sin embargo, paralelo a este cambio se registró un fortalecimiento de actividades extractivas como la minería a cielo abierto, la explotación de hidrocarburos y el agronegocio con impactos socioambientales iguales o peores que en el pasado (Gudynas, 2009). Junto a este aporte, es ineludible aclarar que Gudynas no se inserta en la tradición de Harvey e incluso propone importantes críticas a 
su propuesta analítica, entre las que se destaca el carácter abstracto y excesivamente anclado en lo global, el escaso rol explicativo de la dimensión ecológica, la poca atención al mundo indígena y el limitado abordaje de las alternativas al capitalismo (Gudynas, 2015).

\section{DEL MODELO EXTRACTIVO CONTEMPORÁNEO AL ÁMBITO AGROPECUARIO}

Las nuevas características de la economía global en general y de la explotación de los recursos naturales en particular descritas anteriormente, nos llevan a ahondar en el debate sobre la especificidad de los conceptos para describir las producciones de nuestro continente. Es por ello que se analizarán diversos elementos de la producción de soja en la Argentina para ver cómo y cuánto se inscribe dentro del paradigma del extractivismo. Para ello se situará el análisis en la perspectiva sostenida por Giarracca y Teubal (2013), quienes plantean que el modelo extractivo es constitutivo de la acumulación por desposesión (Harvey, 2004); el mismo se expresa tanto en la actividad minera, la hidrocarburífera y el agronegocio donde la utilización de tecnología de punta, la obtención de rentas a escala global y la competencia con otras actividades económicas adquieren un rol central.

En términos heurísticos, el planteo de Giarracca y Teubal (2013) presenta una serie de dimensiones económicas, políticas y sociales que comparten las actividades extractivas contemporáneas, lo cual permite operacionalizarlas adecuadamente. Como estrategia argumentativa, para el análisis se propone desarrollar dichas dimensiones con elementos relevantes del modelo del agronegocio a fin de fundamentar la hipótesis de trabajo. La mayoría de la información propuesta proviene principalmente de dos fuentes, por un lado, datos estadísticos de organismos nacionales e internacionales y, por el otro, de trabajos académicos conectados con el tema a tratar.

LOCALIZACIÓN TERRITORIAL Y RECURSOS NATURALES

Desde los albores de la producción mercantil existen actividades extractivas — como la minería, la petrolera, la maderera - donde el agotamiento del recurso es inevitable y requieren ubicarse en las inmediaciones de los yacimientos. Sin embargo, en los últimos años la introducción de tecnología ha ampliado los alcances de este modelo. La producción agropecuaria «por naturaleza» requiere del recurso natural tierra/suelo y del agua para riego en algunas regiones. A partir de la «Revolución verde», desde mediados del siglo xx, y posteriormente con la introducción de las semillas transgénicas y la tecnología a ella asociada, la producción a gran escala se expandió.

En Argentina esto se tradujo en un aumento exponencial de la superficie sembrada con soja (ver Figura 1). Si bien los primeros cultivos datan de la década de 1970, claramente el salto más importante se da a partir de 1990/1991, potenciado en la campaña 1996/1997 cuando se libera, en un contexto de precios favorables, la semilla transgénica en el mercado nacional. Entre la campaña 1990/1991 y 1995/1996, la superficie sembrada con soja creció 1.000.00o de hectáreas (ha), en el siguiente periodo (1995/1996 y 2000/2001) se expandió 4.660.00o ha y en el primer quinquenio de la década del 2000 el incremento fue similar: 4.700.00o ha más sembradas con soja. Finalmente, en las últimas campañas entre 2005/2006 y 2011/2012 la expansión superó los 
Figura 1. Superficie implantada por cultivo a nivel nacional (millones de hectáreas)

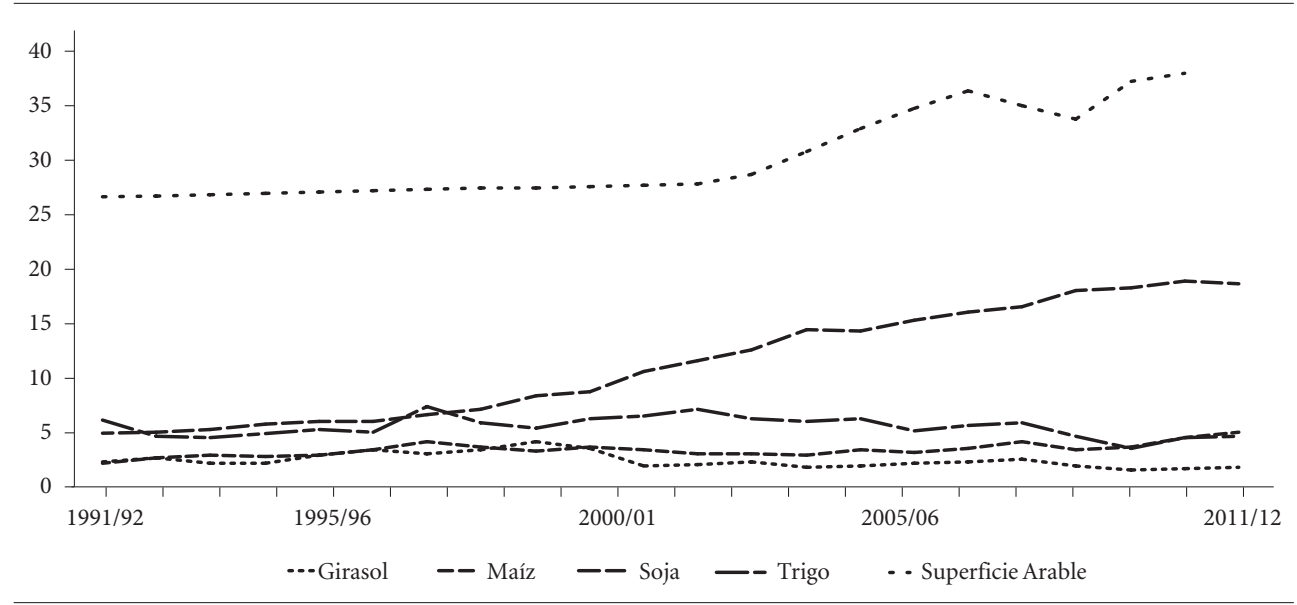

Fuente: Ministerio de Agroindustria de la Nación (MA) y Organización de las Naciones Unidas para la Alimentación y la Agricultura (FAO).

3.000.000 ha, llegando casi a los 3,5 millones si consideramos la campaña 2010/2011. Gracias a estos incrementos, en 2009/2010 la superficie sembrada con soja alcanzó las 18.343.272 ha, lo cual significa que el 49,64\% de la tierra arable del país.

Desde inicios de la década de 1990 la superficie destinada al maíz y al girasol — los principales competidores naturales de la soja- no varió en demasía. No obstante, entre las campañas 1998/1999 y 2000/2001, el girasol redujo su superficie en más de 2.000.00o ha y en los dos últimos períodos mostró una caída de más de un millón de hectáreas para ubicarse en 1.542.945 ha cultivadas. Esto lleva a pensar que la soja avanzó principalmente sobre campos destinados a otros usos pues el crecimiento a nivel nacional que ha tenido entre 1990/1991 y 2009/2010 es de más de 13.000.00o ha. Durante la década de 1990 la extensión del cultivo de soja se realizó principalmente en las tierras agrícolas, hecho que se comprueba con un leve crecimiento de la superficie arable total. Sin embargo, hacia la década del 2000 comenzó un avance de la frontera agrícola a nuevos territorios antes dedicados a la ganadería y/o conservados como bosques nativos.

\section{IMPULSO EN EL MARCO DEL NEOLIBERALISMO ECONÓMICO}

A partir de la década de 1970, los organismos de crédito internacionales y diversos think tanks alineados con el Consenso de Washington, adquirieron una fortaleza inusitada que construyó una hegemonía notable en diversos aspectos del mundo social. Entre ellos, puede destacarse el fortalecimiento de los actores mercantiles privados en el control de la economía, el debilitamiento de las estrategias redistributivas del ingreso y su reemplazo por políticas focalizadas, las medidas de ajuste y la resignación frente al desempleo o la precarización laboral. Además el Estado como macroactor sufrió una notable interpelación que lo criticaba monolíticamente por su ineficiencia. Con este argumento no sólo se restringieron sus roles productivos, sino 
que también se justificaron los procesos privatizadores de las empresas públicas. ${ }^{2}$ Pero definitivamente el Estado no se «achicó», sino que redirigió sus objetivos en función de la acumulación de los nuevos sectores dominantes.

Junto con las privatizaciones se produjo una expansión de la producción de recursos naturales impulsada por transformaciones muy importantes del orden global. Según datos del sistema estadístico de la Comisión Económica para América Latina (CEPAL) procesados para este trabajo, entre 1990 y 1999 el índice de producción agrícola de Argentina subió un 33\%, y entre 1995 y 1999 la producción de plata creció un 54,17\%, la de oro un 3800\%, la de gas natural un 50\% y la de petróleo crudo un 14,37\%. Aun cuando el proceso era incipiente, desde el Estado se emitieron políticas que permitieron la consolidación del modelo extractivo como respuesta al creciente poder de grandes empresas y organismos internacionales. En Argentina, el decreto de desregulación económica de 1991 eliminó la mayoría de los organismos reguladores de las actividades agropecuarias y con ellos desaparecieron los precios sostén, los cupos de producción y el control, al menos parcial, del comercio de granos. Asimismo, la liberación de la soja transgénica en 1996 permitió el despliegue de nuevas formas productivas basadas en tecnologías relativamente novedosas que combinaron transgénesis, agroquímicos y moderna maquinaria diseñada para grandes extensiones. A esto se sumó la creciente importancia del mundo financiero que logró captar y avanzar sobre diversos bienes naturales bajo la figura de los commodities, altamente influidos por la dinámica y los climas bursátiles (Lilliston y Ranallo, 2011; Schumann, 2014).

\section{VINCULACIÓN CON EL INTERÉS DE GRANDES CORPORACIONES}

Desde la década de 1990, en la economía argentina se intensificó la concentración y el poder de las grandes empresas. Tal es así que Schorr, Manzanelli y Basualdo mencionan que la facturación de las 200 empresas más importantes de Argentina pasó de representar el 16,4\% del Valor Bruto de la Producción Nacional en 1993 a 22,8\% en 2001 y 27,1\% en el año 2010 (2012, p. 11). El mismo trabajo señala el ascenso de las compañías vinculadas a la producción y exportación de recursos naturales - granos, oleaginosas, manufacturas de origen agropecuario y minerales - tales como Minera Alumbrera, Minera Argentina Gold, ADM, Alfred Toepfer, Noble, Aluar, Dow Argentina.

Durante gran parte de la década del 2000 también aumentó el peso de las comercializadoras de granos, cuyo sector era controlado por un puñado de empresas multinacionales. En 2008, siete compañías concentraban el 83,5\% del total de los porotos de soja exportados; el 82,8\% del comercio de aceite de soja quedó en manos de cinco firmas y el 89,34\% de los derivados de la oleaginosa fueron vendidos al exterior por solo seis empresas (ver Tabla 1). Si trasladamos estos números al total del comercio internacional de Argentina, las doce primeras empresas centralizaron el $28,21 \%$ del total exportado durante 2008 . La primacía de las empresas multinacionales también tuvo lugar en las procesadoras de alimentos y los canales de distribución, contribuyendo al aumento de la concentración industrial y a la formación de conglomerados; la integración y el oligopolio en la distribución final; la extranjerización y el aumento del control de distintas etapas de la producción a partir de la integración vertical (Teubal y Rodríguez, 2002; Teubal, Domínguez y Sabatino, 2005). 
Tabla 1. Exportaciones de soja y sus derivados por las principales exportadoras argentinas (2008)

\begin{tabular}{lccc}
\hline NOMBRE DE LA EMPRESA & POROTO (\%) & ACEITE (\%) & PELLETS (\%) \\
\hline Cargill S. A. & 19,5 & 27,6 & 21,9 \\
Bunge Argentina S. A. & 10,5 & 16,2 & 18,0 \\
LDC Argentina S. A. (Dreyfus) & 10,2 & 15,2 & 13,5 \\
Aceitera General Deheza S. A. (AGD) & 0,0 & 13,8 & 16,5 \\
Vicentín SAIC & 0,0 & 4,3 & 9,4 \\
Molinos Río de la Plata & 0,0 & 10,1 & 10,2 \\
Asociación de Coop. Argentinas Coop. Ltda. & 5,4 & 2,6 & 2,2 \\
Nidera S. A. & 10,0 & 3,0 & 2,4 \\
Noble Argentina S. A. & 12,0 & 0,0 & 0,0 \\
Oleaginosa Moreno Hermanos & 0,4 & 1,6 & 1,8 \\
A. C. Toepfer International Argentina S. R. L. & 10,1 & 0,0 & 0,0 \\
ADM Argentina S. A. & 11,2 & 0,2 & 0,0 \\
Molino Cañuelas SACIFIA & 1,1 & 0,5 & 0,4 \\
Serv. Integrados para Exportación Agroalimentos & 2,0 & 0,0 & 0,1 \\
Agricultores Federados Argentinos S. C. L. & 0,0 & 0,7 & 0,7 \\
Compañ́a Agroindustrial La Oriental S. A. & 0,1 & 0,0 & 0,0 \\
Otras & 7,5 & 4,2 & 3,1 \\
Total & 100,0 & 100,0 & 100,0 \\
\hline
\end{tabular}

Fuente: Teubal y Palmisano (2010, p. 208).

Otra característica importante de la lógica del agronegocio es la intensificación de las estrategias de integración vertical y horizontal de los establecimientos más importantes. El rol del capital foráneo se incrementó principalmente en dos sectores. Por un lado, la provisión de insumos - semillas, fertilizantes, herbicidas - se convirtió en una sección clave donde las empresas ingresaron a medida que el agronegocio se extendía ofreciendo los adelantos tecnológicos de la ingeniería genética. Asimismo, varias empresas nacionales del área desplegaron un proceso de transnacionalización que se manifestó notablemente en los eslabones de transformación y comercialización alimentaria. Aquí la mayoría de las compañías nacionales recibieron capitales del exterior que reconfiguraron su paquete accionario a la par que se intensificaba la concentración en la producción y comercio de los alimentos en el mercado interno y externo (Teubal y Rodríguez, 2002). En el caso de la producción agropecuaria, el ingreso de capital extranjero fue un poco más limitado y en la mayoría de los casos se originó producto de fusiones y emisión de acciones en diversas bolsas de valores del mundo. Tal es el caso de empresas como Los Grobo, El Tejar o Cresud, que en su estrategia de crecimiento han comprado acciones y empresas diversas a la vez que han puesto en oferta pública parte de su paquete accionario en distintas bolsas de valores como la de Buenos Aires, San Pablo o Nueva York. 


\section{ESCALAS DE PRODUCCIÓN MUCHO MAYORES A LAS TRADICIONALES}

En este sentido se destaca la aparición de nuevas estructuras empresariales que han ganado protagonismo, principalmente por su extensión y el volumen de sus operaciones. Si bien en Argentina siempre existieron grandes empresas y propietarios que controlaban importantes superficies, el avance de estos nuevos actores - fondos de inversión, pooles de siembras $^{3}$ y grandes empresas agropecuarias - parece afectar principalmente a los pequeños y medianos productores. Frente a esta competencia, se dieron múltiples trayectorias que van desde el desplazamiento de la producción y el arrendamiento de las tierras - en el caso que el productor sea propietario y haya sobrevivido a los procesos de endeudamiento de las décadas pasadashasta la ampliación de la escala para poder competir; desde el abandono completo de la actividad agropecuaria a la incorporación subalterna de los productores al modelo convertidos en prestadores de servicios y contratistas.

Los datos censales disponibles en Argentina solo nos permiten analizar los impactos del agronegocio en la década de 1990 al comparar el Censo Nacional Agropecuario (CNA) de 1988 y 2002, ya que el relevamiento realizado en 2008 adolece de numerosas falencias reconocidas por el Instituto Nacional de Estadística y Censos (INDEC, 2009). Al analizar dicho período se observa una notable disminución del número de explotaciones agropecuarias (EAP) en general. Tal es así que mientras en el primer relevamiento se registraron 378.347 EAP con límites definidos, para 2002 ese número cayó a 297.425, significando una merma del 21,39\%. Esta retracción en el número de explotaciones no afectó a todos los estratos por igual. De hecho, siguiendo la estratificación propuesta por Teubal et al. (2005), entre ambos censos hay una caída en términos absolutos y relativos de las pequeñas explotaciones (menos de 200 ha). Junto a ello se dio una reducción en términos absolutos de las explotaciones medianas - entre 201 y 1000 ha- que fue matizada por un incremento en su peso relativo del 2,53\% y una subida tanto en términos porcentuales como cuantitativos de las explotaciones de más de 1000 ha. En lo que respecta a la cantidad de hectáreas controladas por cada uno de los estratos, la tendencia se repite con el lógico agravante que la superficie de las explotaciones medianas también se reduce a favor de las más extensas (ver Tabla 2).

Estos datos muestran claramente el crecimiento de las escalas productivas. Particularmente, para que la producción de soja sea rentable se requiere una importante extensión de tierras. Tal es así que las estimaciones de costos estructurales realizadas por el Ministerio de Agricultura, Ganadería y Pesca de Argentina tienen como base una explotación de entre 500 y 600 ha, lo cual contrasta con el hecho de que para 2002 el 83\% de las EAP del país tenían menos de 500 ha.

\section{USO DE TECNOLOGÍAS DE PUNTA}

Tras la Segunda Guerra Mundial, la extensión de la mencionada «Revolución verde», y años más tarde la difusión de los organismos genéticamente modificados, implicó un ascenso notable de la influencia de las grandes empresas proveedoras y productoras de tecnología. Tal es así que en 2012 el mercado mundial de semillas, pesticidas y fertilizantes estaba controlado por poco más de veinte corporaciones (Gura y Meienberg, 2013). 
Tabla 2. Cantidad de EAP por estratos y su peso relativo a nivel nacional

\begin{tabular}{lrrrr}
\hline \multirow{2}{*}{ TAMAÑo EXPLOTACiONes } & \multicolumn{2}{c}{ CNA 1988 } & \multicolumn{2}{c}{ CNA 2002 } \\
\cline { 2 - 5 } & \multicolumn{1}{c}{ EAP } & \multicolumn{1}{c}{ EAP } & \multicolumn{1}{c}{$\%$} \\
\hline Pequeñas (menos de 200 ha) & 206.736 & 74,5 & 206.736 & 69,5 \\
Medianas (entre 201 y 1000 ha) & 61.652 & 18,2 & 61.652 & 20,7 \\
Grandes (más de 1000 ha) & 29.037 & 7,3 & 29.037 & 9,8 \\
Total & 297.425 & 100,0 & 297.425 & 100,0 \\
\hline
\end{tabular}

Fuente: Elaboración propia con datos del Instituto Nacional de Estadística y Censos (INDEC).

En el caso argentino, se observa una combinación de innovaciones organizacionales y tecnológicas. Las primeras reconfiguran quiénes y de qué manera se hacen las tareas, y las segundas se vinculan principalmente con el paquete semilla transgénica/siembra directa/herbicida y la maquinaria asociada a fin de estandarizar la producción y prescindir de los conocimientos locales. La extensión del paquete tecnológico transformó las prácticas del mundo agrario pampeano y argentino a partir de la consigna de que su extensión enfrentaría los procesos de degradación del suelo diagnosticados a finales de los 80. Sin embargo, como se verá adelante, las nuevas tecnologías permitieron procesos de extracción de nutrientes más intensivos para la tierra (Palmisano, 2015).

\section{DESPLAZAMIENTO HUMANO MASIVO}

Hacia mitad de la primera década del siglo xxi un trabajo de Aparicio (2005) advertía que el modelo del agronegocio

no es compatible con una estimulación de la dinámica del mercado de trabajo [... pues] la «pampeanización sojera» en gran parte del país desplaza trabajadores de producciones tradicionales (no sólo campesinos) y los «oasis» modernos y «dinámicos» no reemplazan ni constituyen mercados «estables» de trabajo. (p. 193)

En términos nacionales, desde hace varias décadas el sector agropecuario viene perdiendo trabajadores principalmente a causa de la mecanización de los distintos cultivos (Neiman, 2010). Al considerar los CNA de 1988 y 2002, la variación intercensal de personas que trabajan permanentemente en las EAP cayó un 24,89\% (259.138 personas menos). En el caso de los censos de población de 1991 y 2001, la cantidad de trabajadores vinculados a la agricultura, ganadería, caza y silvicultura bajó un 33,4\% (449.886 trabajadores menos), mientras que la población ocupada total se redujo en un 11,76\%. Para los años posteriores, la información disponible es fragmentaria y no cotejable, de hecho el censo de población realizado en 2010 no relevó de la misma manera las variables de ocupación impidiendo conocer la evolución de la Población Económicamente Activa (PEA) agropecuaria.

Por su parte, los datos periódicos del INDEC se circunscriben a los puestos de trabajo declarados en el sector, que como se indica, sólo tienen en cuenta aquellos trabajadores a los que se le realizan aportes jubilatorios y de cargas sociales. Entre 1995 y 2012 hubo un aumento considerable de trabajadores registrados en la actividad pues se pasó de 136.000 a 345.000. Sin embargo, 
más que un crecimiento del total de trabajadores debe considerarse un incremento en el registro de aquellos que ya trabajaban allí. Solo así podríamos explicar como la cantidad de trabajadores registrados aumenta a lo largo de la década de 1990 mientras la variación entre los censos nacionales arroja una caída del $33,4 \%$ de la población económicamente activa (PEA) del sector.

En el caso de la provincia de Buenos Aires, donde pueden hallarse más datos, el censo nacional de 1991 registró 252.955 personas mayores de 14 años ocupadas en el sector, y para 2001 solo quedaron 169.712, es decir, hubo una caída sectorial del 32,91\% mientras que la PEA total de la provincia cayó 19,72\%. En caso de comparar los CNA de 1988 y de 2002 la reducción de los trabajadores permanentes bonaerenses es del 34,44\%. Paralelamente se consolidó el proceso de asalarización de los trabajadores rurales, cuyo porcentaje sobre la PEA del sector ascendió en 2001 al $55 \%$. Estos valores pueden leerse como un indicio de deterioro de la organización familiar de la producción agraria típica de Argentina asentada en una «relación social de trabajo» (Du Tertre, 1997) que une tecnologías, recursos vivos y un «espacio rural» que se extiende a los trabajadores familiares no asalariados. En lo que respecta a los prestadores de servicios agropecuarios - contratistas- que florecieron en paralelo al modelo del agronegocio, podemos afirmar que la cantidad de trabajadores que empleó estuvo lejos de revertir la tendencia que se dio durante la década de 1990. Tal es así que según la Encuesta Provincial de Servicios Agropecuarios realizada en la provincia de Buenos Aires, hay un crecimiento de los trabajadores en el sector entre 2002 y 2006 del 64,27\% pasando de 15.517 empleados a 25.490 . Este valor está lejos de revertir la desaparición de 83.243 puestos de trabajo agropecuarios que se registró entre los censos 1991 y 2001.

\section{ALTO CONSUMO DE RECURSOS NO REPRODUCIBLES}

La extracción de nutrientes del suelo es el indicador más claro de uso del recurso tierra. Con la expansión territorial y la tendencia al monocultivo que ha tenido la soja y la intensificación de la producción agraria en general, la extracción de nutrientes se ha vuelto mayor a la repuesta afectando la fertilidad de los suelos. Según un trabajo de Cruzate y Casas (2012) en la campaña agrícola argentina de 2010/2011 solo se repusieron el 34,6\% de los nutrientes —fósforo, calcio, nitrógeno, potasio y azufre - a la vez que la materia orgánica del área núcleo pampeana sufrió un descenso promedio de $0,5 \%$ en las últimas tres décadas. Las zonas de mayor extracción de nutrientes coinciden con las más fértiles del país y el trabajo afirma la

existencia de sistemas productivos que no son sostenibles, afectando negativamente los niveles de fertilidad e incrementado los procesos de degradación de los suelos y, por ende, limitando el crecimiento de la producción agrícola nacional. (Cruzate y Casas, 2012, p. 14) ${ }^{4}$

Esto implica que las áreas más fértiles son aquellas que mayor presión reciben sobre los nutrientes del suelo. La degradación queda solapada, pues las mermas en el rendimiento por hectárea, que solían utilizarse como índice del deterioro, se revierten a partir de la capacidad de los nuevos materiales genéticos para captar nutrientes de la tierra y aumentar la presión sobre el suelo. Así, esta mayor productividad se da a costa de un aumento en la exportación de nutrientes.

Otro punto vital que se debería considerar gira en torno a la utilización y exportación de agua detrás de la producción agropecuaria en general y la sojera en particular. En términos analíticos, la unidad de medida de este proceso es el agua virtual, entendida como «la cantidad 
de agua por unidad de alimento que es o que podría ser consumido durante su proceso de producción» (Food and Agriculture Organization [FAO], 2003, p. 28). Según Dalin et al. (2012), entre 1986 y 2007 el comercio de agua virtual se incrementó en un 118\% y a partir de la década del 2000 Sudamérica se convirtió en el mayor exportador del mundo. Esto se dio en paralelo al ascenso de China como el primer importador a nivel global con el 13\% del comercio mundial de agua virtual. Según los autores citados, la primacía del país asiático se debió al aumento de las exportaciones de productos basados en soja que fue cubierto casi en su totalidad por solo tres naciones: Argentina, Brasil y Estados Unidos. Respectivamente exportaron a China el 28\%, 37\% y $33 \%$ de los 71 millones de $\mathrm{m}^{3}$ de agua virtual. Para Argentina este valor implicó el 40\% del agua virtual que se exportó en granos. Para 2010 el balance del comercio de agua virtual nacional mostró que en forma de granos se vendieron casi 46.000 millones de $\mathrm{m}^{3} \mathrm{y}$ se importaron solo 3100 millones (INTA, 2012).

Si bien esto no implica una pérdida efectiva del recurso por la dinámica propia del «ciclo del agua», el comercio de agua virtual pone en evidencia la intensificación de relaciones comerciales asentadas en la abundancia de recursos naturales a la que los países latinoamericanos parecen estar condenados. Si bien la sobreexplotación del agua en la Argentina parece un fenómeno de difícil aseveración, es importante considerar los impactos sobre la tierra ligados al uso intensivo del recurso, tales como la «intrusión salina, salinización, perdida de estructura del suelo, lavado de nutrientes, contaminación» (Pengue, 2006, p. 61).

\section{ORIENTACIÓN HACIA LAS EXPORTACIONES}

Quizá este sea uno de los aspectos principales del modelo en tanto se registra una renovada orientación exportadora de gran parte de la producción agropecuaria. Por poner un solo ejemplo, el Anuario estadístico 2012 de la CEPAL muestra en la última década un crecimiento de la participación de las exportaciones primarias con respecto al total en casi todos los países latinoamericanos y sus bloques económicos (CEPAL, 2012). El caso de Argentina es particular, pues luego de un aumento en los primeros años de la década, cuyo punto más alto se dio en 2003 cuando las exportaciones primarias representaban un $72,2 \%$ del total, se registró una retracción de 3 o 4 puntos porcentuales y una posterior recuperación en 2011 (CEPAL, 2012).

Específicamente la soja ha tenido un rol central en la exportación. Según la FAO, en 2011 la producción de soja en Argentina alcanzó los 48,88 millones de toneladas y las exportaciones en concepto de porotos, tortas y aceites sumaron 42,07 millones de toneladas que representan el 86,07\% de la producción. Asimismo, si se considera que el proceso de extracción de aceite de soja tiene una merma de entre un 3 y el $9 \%$, el total de la producción orientada a la exportación debe corregirse para incorporar esta pérdida como parte de la cadena de exportación. Por tanto, si consideramos una merma del 3\%, el 88,03\% de la producción está orientada al exterior, mientras que si este valor se fija en el 9\%, se exporta el 92,39\% de la soja.

La participación de Argentina no solo se manifiesta a nivel interno, sino también en el contexto global. Siguiendo con información de la FAO, en 2012 Argentina fue el tercer productor mundial de porotos de soja concentrando el 20,34\% del volumen mundial, apenas por debajo de Brasil (25,95\%) y Estados Unidos (34,41\%). En el caso de la producción de aceite de soja, Argentina también ocupó el tercer lugar con el 17,26\%, por detrás de Estados Unidos (20,8\%) y 
China (24,44\%). Asimismo, fue el principal exportador internacional de harinas y aceites derivados de la oleaginosa, centralizando para 2011 el 41,33\% y 44,2\% del mercado respectivamente. La importancia de la soja en los balances comerciales argentinos comenzó a evidenciarse en 2001 cuando la participación del complejo sojero - poroto, aceite, pellets y otros derivados - en las exportaciones nacionales superó el $17 \%$ para llegar a un pico de $24,4 \%$ en 2007 . Los dos años siguientes dicho valor se mantuvo por encima del 23\% y en 2009 representó el 24,8\% de las exportaciones nacionales (ver Tabla 3). Esto implicó que poco menos de un cuarto de las exportaciones argentinas se vinculó con este cultivo cuya aparición en la economía argentina es bastante reciente. La orientación exportadora es tan importante en Argentina que, según datos la FAO, la cantidad de kilocalorías diarias por persona en 2011 - que representan el alimento disponible en el mercado interno- era similar a 1996, algo que resulta paradójico si consideramos que la producción de granos entre los mismos años creció más de un 150\% (Teubal y Palmisano, 2013).

\section{CONFLICTIVIDAD CON LAS ACTIVIDADES TRADICIONALES}

Varios de los puntos señalados anteriormente ponen en evidencia la difícil convivencia entre los agronegocios y otro tipo de actividades y modos de vida. Principalmente, la voracidad por la ocupación territorial ha llevado al desplazamiento de numerosas poblaciones de todos los rincones del país. En el caso de las regiones extrapampeanas ${ }^{5}$ se ha registrado un arrinconamiento de los cultivos industriales y la puesta en producción agrícola de yungas, monte nativo y territorios de comunidades campesinas e indígenas ubicados, principalmente en la zona norte del país (GEPSYD, 2010 y GER, 2004). A las estrategias de arrinconamiento silencioso o de violencia abierta para la expulsión de campesinos, le siguen las topadoras que desmontan la vegetación nativa para poder sembrar soja o pasturas para la ganadería desplazada desde zonas más fértiles.

En la región pampeana, y particularmente en la provincia de Buenos Aires, la ganadería y la producción de leche fueron las más afectadas. Según datos del MA, para la campaña 2002/2003 la superficie bonaerense cultivada con soja fue de 2,47 millones ha ( $23 \%$ del total implantado) y para 2012/2013 ese valor había alcanzado los 6,73 millones ha, lo que implicó un crecimiento del 172,5\%. En simultáneo se registró un marcado ritmo de cierre de establecimientos productores de leche - tambos- a la par de un sostenimiento de la producción. Entre 1988 y 2008 cerraron sus puertas el 62,8\% de los tambos bonaerenses, a un ritmo de 385 por año (ver Tabla 4). Entre 2008 y 2010 hubo un descenso del rodeo vacuno nacional de más de 8,5 millones de cabezas, que implicó una merma de casi el 15\% del total nacional. Paralelamente se extendió un tipo de producción ganadera intensiva: los feedlots. Estos establecimientos se dedican al engorde confinado de los vacunos con alimentos balanceados. Esta técnica es complementaria a la expansión sojera pues permite el reemplazo de la alimentación a campo en pasturas por un sistema que requiere menores extensiones. Para 2010 había unas 470 empresas nucleadas en la Cámara Argentina de Feedlots con una capacidad de encierre de 1.000.00o de cabezas y una producción anual en 2009 de 2,5 millones de cabezas, las cuales representan un 15,57\% del total de vacunos faenados en ese año (Cámara Argentina de Feedlots, 2010).

Los datos presentados muestran la expansión de este «nuevo» sistema de producción ganadera que puede ubicarse claramente en el registro del agronegocio, tanto por su compatibilidad con el avance sojero como por sus propias características. La principal cualidad que se 
Tabla 3. Exportaciones de productos vinculados al complejo sojero en relación con las exportaciones totales.

\begin{tabular}{lcccc}
\hline PERÍODO & POROTOS DE SOJA & ACEITES DE SOJA & HARINAS DE SOJA & COMPLEJO SOJERO \\
\hline 1997 & 0,55 & 3,95 & 7,73 & 12,23 \\
1998 & 2,46 & 5,59 & 6,59 & 14,64 \\
1999 & 2,19 & 5,38 & 7,72 & 15,29 \\
2000 & 2,95 & 3,58 & 8,24 & 14,77 \\
2001 & 4,69 & 3,98 & 9,05 & 17,71 \\
2002 & 4,36 & 5,26 & 10,01 & 19,63 \\
2003 & 6,16 & 6,96 & 10,91 & 24,03 \\
2004 & 5,03 & 6,76 & 10,43 & 22,22 \\
2005 & 5,68 & 5,56 & 9,41 & 20,65 \\
2006 & 3,82 & 5,99 & 9,37 & 19,19 \\
2007 & 6,16 & 7,92 & 10,31 & 24,39 \\
2008 & 6,47 & 6,91 & 10,09 & 23,47 \\
2009 & 3,03 & 5,90 & 14,57 & 23,50 \\
2010 & 8,53 & 5,21 & 11,07 & 24,80 \\
2012 & 3,8 & 5,14 & 12,55 & 21,49 \\
\hline
\end{tabular}

Fuente: Elaboración propia a partir de datos del INDEC.

Tabla 4. Cantidad de tambos bonaerenses por cuenca

\begin{tabular}{lrrrr}
\hline CUENCA & 1988 & 1996 & 2000 & 2008 \\
\hline Abasto Sur & 1.924 & 1.240 & 702 & 513 \\
Abasto Norte & 1.239 & 752 & 445 & $\mathrm{~s} / \mathrm{d}$ \\
Oeste & 2.505 & 2.112 & 1.621 & $\mathrm{~s} / \mathrm{d}$ \\
Mar y Sierras & 459 & 305 & 312 & $\mathrm{~s} / \mathrm{d}$ \\
Cuenca Sur & 207 & 105 & 145 & $\mathrm{~s} / \mathrm{d}$ \\
Fuera de Cuenca & 413 & 185 & 26 & $\mathrm{~s} / \mathrm{d}$ \\
Total & 6.747 & 4.699 & 3.251 & 2.508 \\
\hline
\end{tabular}

Fuente: Elaboración propia en base a datos del Ministerio de Asuntos Agrarios de la Provincia de Buenos Aires.

esgrime para el sistema es la intensificación, pues en muy pocas hectáreas es posible realizar el engorde que en condiciones tradicionales hubiese necesitado de grandes extensiones. Al mismo tiempo, los costos laborales no suben en demasía pues solo es necesaria una persona cada 500 o 1000 animales en engorde. A estas observaciones, los feedlots suman otras falencias: contaminación ambiental, malestar animal, merma en la calidad de la carne producida suelen ser las más nombradas (García, 2002; Portillo y Conforti, 2009). En contraste, la carne destinada a la exportación de alta gama a Europa (conocida como cuota Hilton) tiene como requisito que los animales sean alimentados exclusivamente en pasturas, por lo que la carne proveniente de los establecimientos con engorde a corral es destinada principalmente al consumo interno. 
PODEMOS VIVIR SIN ORO NI SOJA, PERO NO SIN AGUA NI ALIMENTOS

Mencionamos anteriormente el carácter meramente exportable que tiene para Argentina la soja, aun cuando en los últimos años el consumo de aceite de soja haya subido en comparación con el de girasol. Según datos de la FAO, el suministro de aceite de soja per cápita para alimentación en 2009 fue de 2,7 kg, mientras que el de aceite de girasol fue de 9,8. En comparación con 2001 esto implicó un aumento del 3,9\% en el suministro de aceite de soja y una caída del 10,9\% del aceite de girasol. Sin embargo, el consumo total nacional de aceite de soja ha crecido a causa de la expansión del biodiesel. Tal es así que en 2013 Argentina consumió el 33,25\% del aceite de soja producido de lo cual el 93,36\% se utilizó para producir biodiesel (Calzada, 2014). En definitiva, en términos de la dieta de los argentinos, la soja tiene una participación muy escasa a pesar del aumento de su uso en alimentos procesados a través de la lecitina.

Por otro lado, es importante aclarar que el carácter no esencial de los productos del extractivismo debe considerarse en el marco de las propias dinámicas de producción que el modelo extiende. Es probable que la soja sea fundamental para la dieta de otras culturas a lo largo del mundo y la oleaginosa per se no debería sacrificarse como una opción de consumo siempre y cuando se produzca de manera ambientalmente sustentable. En este sentido, nos parece fundamental plantear el carácter no esencial del modelo del agronegocio en general y del sojero en particular para la Argentina y para el mundo. En un texto ampliamente documentado Altieri y Nicholls (2000) desarman los argumentos que suelen utilizarse para imponer la agricultura industrial a gran escala (agronegocio en los términos de este trabajo) por sobre otras formas productivas agrarias. Los autores destacan los impactos negativos de estas técnicas a los que pueden sumarse los efectos que genera también la difusión de variedades transgénicas. En esta línea es importante dejar claro que existen alternativas productivas al modelo del agronegocio asentadas en formas distintas de organizar la producción. De ahí que su existencia sea una característica de la configuración políticoeconómica del sector agropecuario más que una condición esencial para el abastecimiento alimentario mundial.

Regresando al ámbito argentino, suele argumentarse que el peso de los ingresos por derechos de exportación es muy importante para las arcas fiscales. Aunque su participación ha crecido, está lejos de ubicarla entre las fuentes de recursos públicos más importantes. Según un análisis de la recaudación nacional (Teubal y Palmisano, 2010), la participación de los derechos de exportación subió a partir de 2007, aunque la recaudación por semillas, aceites y derivados se mantuvo en promedio apenas por encima del $6 \%$ de los ingresos fiscales totales. En contraste, la fuente de ingresos más importante en la década del 2000 siguió siendo el Impuesto al Valor Agregado, una tasa indirecta que erogó en el período 26\% de la recaudación. En definitiva, los derechos de exportación, como mecanismo de captación de la renta, tuvieron una importancia relativa en los ingresos del Estado.

\section{MUCHO VALOR DE CAMBIO, MUY POCO VALOR DE USO}

Marx (2006) afirma que toda mercancía tiene un valor de uso y un valor de cambio. El primero es la capacidad de un objeto para satisfacer una necesidad puntual y está determinada por las características propias del objeto y el mundo de necesidades históricamente determinadas en 
el cual se inserta. El valor de cambio, por su parte, está representado por la cantidad de trabajo abstracto o tiempo de trabajo socialmente necesario para producir cada mercancía.

En el caso de las actividades extractivas, este punto se justifica al considerar el carácter meramente mercantil — en realidad, en cuanto commodity — que tiene la soja, pues la comunidad solo utiliza el producto de manera marginal como resultado de un avance del modelo más que por seguimiento de pautas de consumo culturales. De hecho, la proporción de la producción que se destina a recomponer el ciclo productivo del commodity es muy pequeña. ${ }^{6}$ En términos de procesamiento y comercio de la soja, se vio que aproximadamente el $90 \%$ de la producción se destina al mercado externo y la restante se utiliza como suplemento para el engorde de animales confinados y en el caso del aceite de soja para producir biodiesel. En síntesis, en el caso del complejo sojero, el valor de cambio es el que prima por sobre el uso que se le da al cultivo en las regiones donde se produce.

\section{GENERACIÓN DE GRANDES «DESECONOMÍAS» EXTERNAS}

Frente al enorme caudal de renta que genera el modelo a raíz de su vínculo con el sector externo aparecen los valores no contabilizados que se convierten en deseconomías externas. Este concepto esconde los «costos» sociales, económicos, ambientales y referidos a la salud humana que genera el modelo del agronegocio. Probablemente su importancia sea tanta que su solo cálculo puede poner en cuestionamiento el modelo en su conjunto. Una primera cuestión que se debe considerar tiene que ver con los peligros que entraña una excesiva especialización en un determinado cultivo, por ejemplo, la soja, la cual por definición dificulta el desarrollo de toda biodiversidad, producción de otros cultivos o alimentos más importantes desde el punto de vista de las necesidades de la población en su conjunto, afectando también la necesaria rotación de los cultivos y producción pecuaria para mantener aunque sea mínimamente la sustentabilidad del sector.

El segundo tópico que destacar es el avance sobre los bosques nativos. Hacia 1987 había poco más de 35,1 millones de ha de bosques en Argentina y para 1998 quedaban 33,1 millones. A partir de allí el ritmo de deforestación aumentó para llegar en el período 1998-2002 a la pérdida de 230.000 ha por año de bosques nativos. Las provincias más afectadas fueron Chaco, Santiago del Estero y Salta. Según datos de la Secretaría de Ambiente y Desarrollo Sustentable de la Nación, entre 1998 y 2002 la provincia de Salta registró una superficie deforestada de 194.389 ha, mientras que entre 2002 y 2006 el desmonte se duplicó hasta llegar a las 414.932 ha (Ortiz et al., 2008). Durante el año 2007, en pleno tratamiento parlamentario de la Ley de Bosques, 7 el gobierno salteño autorizó el desmonte de 523.722 ha, evitando la moratoria que establecía la norma (Montenegro et al., 2004).

En definitiva se destruyeron a nivel nacional 250.000 ha de monte nativo por año que se sumaron al grave proceso de «degradación» de las masas forestales restantes por la extracción de maderas y la carga excesiva de ganado. La deforestación también influye sobre la capacidad de absorción del suelo, ya que el cultivo agrícola no retiene el agua tanto como los ecosistemas boscosos y la misma termina escurriéndose hacia los ríos (Brouver y Manghi, 2006). Al mencionado deterioro ambiental, debemos sumar el hecho que estos bosques son también el territorio de miles de comunidades campesinas, pueblos originarios y criollos, que dependen de 
ellos cotidianamente. Para las comunidades que viven en y de los bosques, éstos constituyen su hogar y su fuente de subsistencia. La tasa de desmonte actual significa su expulsión hacia la pobreza suburbana (Schmidt, 2014; Sili, 2016; Valenzuela, 2005).

En tercer lugar, debemos destacar el uso de plaguicidas en la agricultura. Según datos de la FAO, en 1993 el agro argentino utilizaba $25.835 \mathrm{t}$ de ingredientes activos para herbicidas, fungicidas e insecticidas mientras que para 2010 este valor ascendió a $273.141 \mathrm{t}$, un aumento del 957\%. ${ }^{8}$ Gran parte de esta subida se da por un incremento de casi el $1200 \%$ en el consumo de herbicidas, de los cuales el glifosato es el más difundido. Es importante destacar que en el caso del glifosato existen importantes y documentados estudios que advierten sobre la peligrosidad de este herbicida para la salud humana, lo cual se suma a numerosas denuncias e investigaciones de organizaciones y poblaciones afectadas. ${ }^{9}$

\section{REFLEXIONES FINALES}

El análisis de las múltiples dimensiones propuestas por Giarracca y Teubal permite dar cuenta de los alcances del agronegocio y su inserción en un régimen de acumulación global que impone a los países del Sur global el rol de reservorio y proveedor de materias primas. Las potencias tradicionales o las hegemonías en ascenso, como el caso de China, se abastecen de los commodities de nuestras regiones trasladando a los territorios los impactos socioambientales de su producción. Argentina se ha vuelto uno de los ejemplos más expresivos de los alcances del avance de la lógica extractivista en el sector agrario. Con casi el 50\% de su tierra arable cubierta con soja destinada al mercado mundial, los ingresos económicos surgidos con el ciclo de precios altos de las materias primas no pueden desestimar las enormes deseconomías externas que no son ni más ni menos que formas de nombrar al sufrimiento social.

Por otro lado, la deficiente reposición de nutrientes del suelo nos permite conectar las dinámicas propias del agronegocio sojero con actividades extractivas tradicionales como la minería, otorgándole a la tierra sometida a estas condiciones productivas el estatus de no renovable. Sin embargo, los ciclos de largo plazo que afectan la fertilidad de la tierra como así también estrategias productivas alternativas que pueden revertir procesos como el indicado, obligan a avanzar en un debate en el que no se generalicen los sentidos comunes propuestos por los actores hegemónicos. Asimismo, consideramos de vital importancia ahondar en la proliferación de discursos que acompañaron los distintos momentos del agronegocio sojero, más aún cuando varios de los autores citados asignan un peso importante a esta dimensión y conectándolo directamente con el extractivismo actual.

\section{NOTAS}

1 Principalmente Bonefeld, [2001] 2012; Dalla Costa, [1994] 2004; Luxemburg, 1964; y Mandel, 1980.

2 Ejemplos de esto fueron la Empresa Nacional de Telecomunicaciones (Pierbattisti, 2008) y Aerolíneas Argentinas (Thwaites Rey, 2001). Asimismo, Basualdo (2001) desarrolla el surgimiento del discurso privatizador y sus alcances socioeconómicos.

3 Se entiende por pool de siembra a una organización más o menos formal en el que confluye el dueño de la tierra, un contratista que realiza la labores y un ingeniero agrónomo, que convienen una producción 
aportando cada uno sus recursos y se reparten utilidades de acuerdo al peso asignado a su participación. 4 Otros trabajos que enfatizan sobre la extracción de nutrientes en diversos puntos de Argentina son Heredia, Duffau y Conti (2003); Losinno y Conti (2005); y Ventimiglia, Carta y Rillo (2000).

5 Las zonas extrapampeanas son aquellas que se ubican por fuera región comprendida por la provincia de Buenos Aires, el centro y sur de Santa Fe, gran parte de la provincia de Córdoba, el centro y sur de San Luis y la parte este de La Pampa.

6 Según un informe técnico del INTA (Morel, s/f), se requieren entre 55 y $90 \mathrm{~kg}$ de soja para implantar una hectárea. En la campaña 2011/2012, según datos del MA, se sembraron 18.670.937 hectáreas de soja en el país lo que considerando una media de $70 \mathrm{~kg}$ de semillas por hectáreas daría un total de 1.306.965,6 toneladas destinadas para este fin. La producción de la oleaginosa para dicho período fue de 40.100.197 toneladas, por lo que los porotos destinados a semilla representan poco más del 3\% de la producción total. 7 Nos referimos a la ley 26.331 de Presupuestos mínimos de protección ambiental de los bosques nativos promulgada en 2007 .

8 Datos de elaboración propia a partir de la Dirección de Estadística de la FAO: http://faostat3.fao.org/

9 Véase Ávila Vázquez (2014); Benachour y Séralini (2008); Benachour et al. (2007); Domínguez y Sabatino (2005); GRR (2009); Kaczewer (2002, 2009); Montenegro (2006); Oliva, Spira y Multigner (2001); Paganelli et al. (2010).

\section{REFERENCIAS}

Altieri, M. y Nicholls, C. I. (200o). Agroecología. Teoría y práctica para una agricultura sustentable. México DF, México: Programa de las Naciones Unidas para el Medio Ambiente.

Aparicio, S. (2005). Trabajos y trabajadores en el sector agropecuario de la Argentina. En N. Giarracca y M. Teubal (Comp.), El campo argentino en la encrucijada: estrategias y resistencias sociales, ecos en la ciudad (pp. 193-221). Buenos Aires, Argentina: Alianza.

Ávila Vázquez, M. (2014). Agricultura tóxica y pueblos fumigados en Argentina. Revista + E, 1(4), 28-34.

Banerjee, S. B. (2010). Corporate Social Responsibility: The good, the bad and the ugly. Critical Sociology, 1(34) 51-79.

Basualdo, E. M. (2001). Sistema político y modelo de acumulación en la Argentina. Notas sobre el transformismo argentino durante la valorización financiera. Buenos Aires, Argentina: FLACSO/UNQ/IDEP.

Benachour, N. y Séralini, G-E (2009). Glyphosate Formulations Induce Apoptosis and Necrosis in Human Umbilical, Embryonic, and Placental Cells. Chemical Research in Toxicology, (22), 97-105.

Benachour, N., Sipahutar, H., Moslemi, S., Gasnier, C., Travert, C. y Séralini, G-E (2007). Time- and Dose-Dependent Effects of Roundup on Human Embryonic and Placental Cells. Archives of EnvironmentalContamination and Toxicology, 53(1), 126-133.

Bonefeld, W. (2012). La permanencia de la acumulación primitiva: fetichismo de la mercancía y constitución social. Revista Theomai, (26). Recuperado de http://revista-theomai.unq.edu.ar/ NUMERO\%2026/Bonefeld\%2O-\%2OLa\%2o permanencia\%2ode\%2ola\%2Oacumulaci\%C3\%B3n\%2O primitiva.pdf

Brouver, M. y Manghi, E. (2006). Cambios estructurales en la Selva Tucumano Boliviana entre 1975 y 2000 en la zona de Tartagal. Buenos Aires, Argentina: Dirección de Bosques, Secretaria de Ambiente y Desarrollo Sustentable.

Cafiero, B. (2010). La intervención social de las empresas: reflexiones en torno a la práctica, el discurso y las implicancias de la noción de Responsabilidad Social Empresarial. Economía y Desarrollo, 9(1), 147-167.

Calzada, J. (2014). El clásico Argentina-Brasil pero en la cancha del aceite de soja y el biodiesel. Informativo 
Semanal de la Bolsa de Comercio de Rosario, 23(1664). Recuperado de https://www.bcr.com.ar/ Publicaciones/Informativo\%20 semanal/bcr2014_06_27.pdf

Cámara Argentina de Feedlot (2010) Cámara. Recuperado de http://www.feedlot.com.ar/sitio/?page_id=17 CEPAL (2012). Anuario estadístico de América Latina y el Caribe. Santiago de Chile: Organización de las Naciones Unidas/CEPAL. Recuperado de http://www.cepal.org/publicaciones/xml/2/48862/ AnuarioEstadistico2012.pdf

Cruzate, G. y Casas, R. (2012). Extracción y balance de nutrientes en los suelos agrícolas de la Argentina. Informaciones Agronómicas de Hispanoamérica, (6), 7-14.

Dalin, C., Konar, M., Hanasaki, N., Rinaldo, A. y Rodriguez-Iturbea, I. (2012). Evolution of the global virtual water trade network. Proceedings of the National Academy of Sciences of the United States of America, 109(16), 5989-5994.

Dalla Costa, M. (2004).Capitalism and Reproduction. The Commoner. Recuperado de http://www.commoner.org. uk/o8dallacosta.pdf

Domínguez, D. y Sabatino, P. (2006). Con la soja al cuello: crónica de un país hambriento productor de divisas. En H. Alimonda (Comp.), Los tormentos de la materia. Aportes para una ecología política latinoamericana (pp. 249-274). Buenos Aires, Argentina: CLACso.

Du Tertre, C. (1997). Un enfoque sectorial del trabajo. R. Boyer e Y. Saillard (Ed.), Teoría de la regulación: estado de los conocimientos (pp. 135-142). Buenos Aires, Argentina: Oficina de publicaciones del cBC, vol. 2 .

FAO (2003). Descubrir el Potencial del Agua para la Agricultura. Roma, Italia: FAO. Recuperado de http:// www.fao. org/docrep/oo6/y4525s/y4525soo.htm\#Contents

García, P. T. (2002). Factores de riesgo. Revista IDIA XXI, (2), 195-199.

GEPCyD (2010, agosto). La violencia rural en la Argentina de los agronegocios: crónicas invisibles del despojo. Ponencia presentada en vi Jornadas de Antropología Social. Buenos Aires, Argentina.

GER (2004). Desalojos y arrinconamientos de campesinos y comunidades indígenas en la Argentina. Realidad Económica (203), 111-120.

Giarracca, N. y Teubal, M. (2008). Del desarrollo agroindustrial a la expansión del «agronegocio»: el caso argentino. En B. Mançano Fernandes (Org.), Campesinato e agronegócio na América Latina: a questão agraria atual (pp.139-164). San Pablo, Brasil: Expressão Popular.

Giarracca, N. y Teubal, M. (2013). Las actividades extractivas en Argentina. En N. Giarracca y M. Teubal (Coord.), Actividades extractivas en expansión ¿Reprimarización de la economía argentina? (pp. 19-43). Buenos Aires, Argentina: Antropofagia.

GRR (2009). Pueblos Fumigados. Buenos Aires, Argentina: GRR.

Gudynas, E. (16 de octubre de 2015). Debate Gudynas/Harvey: La necesidad de romper con un «colonialismo simpático» (con reacciones). Fundación Rosa Luxemburgo. Recuperado de http://rosaluxspba.org/es/la-necesidad-de-romper-con-un-colonialismo-simpatico/

Gudynas, E. (2009). Diez tesis urgentes sobre el nuevo extractivismo. Contextos y demandas bajo el progresismo sudamericano actual. En AA.vv., Extractivismo, política y sociedad (pp. 187-225). Quito, Ecuador: CAAP/ CLAES.

Gura, S. y Meienberg, F. (2013). Agropoly - A handful of corporations control world food production. EcoNexus. Recuperado de http://www.econexus.info/publication/agropoly-handful-corporations -control-world-food-production 
Harvey, D. (2004). The New Imperialism: Accumulation by Dispossession. Socialist Register, (40), 99-129. Recuperado de http://biblioteca.clacso.edu.ar/clacso/se/20130702120830/harvey.pdf

Headey, D. y Fan, S. (2010). Reflections on the Global Food Crisis. How Did It Happen? How Has It Hurt? And How Can We Prevent the Next One? Washington DC, USA: International Food Policy Research Institute. Heredia, O.; Duffau, V. y Conti, M. (2003). Cambios de la Fertilidad Edáfica en Suelos de la Región Pampeana. Informaciones Agronómicas de Hispanoamérica, (19). Recuperado de http://www. ipni.net/publication/ia-lacs.nsf/o/5236C5AB4F61F9EF852579990061D278/\$FILE/HerediaCambiosFertilidadRegPampeana.pdf

INDEC (2009). Censo Nacional Agropecuario 2008-CNA'08. Resultados provisorios. INDEC. Recuperado de http:// www.indec.gov.ar/nuevaweb/cuadros/novedades/_10_09.pdf

INTA (13 de marzo, 2012). Huella hídrica: eficiencia en el uso del agua. Inta Informa. Recuperado de http:// intainforma. inta.gov.ar/?p=12694

Kaczewer, J. (2002). Toxicología del glifosato: riesgos para la salud humana. Buenos Aires, Argentina: Universidad de Buenos Aires.

Kaczewer, J. (2009). Uso de agroquímicos en las fumigaciones periurbanas y su efecto nocivo sobre la salud humana. Biodiversidadla. Disponible en http://www.biodiversidadla.org/Objetos_ Relacionados/file_folder/archivos_word_2/ UsO_DE_AGROQUIMICOS_EN_LAS_FUMIGACIONES_ PERIURBANAS_Y_SU_EFECTO_NOCIVO_SOBRE_LA_SALUD_HUMANA

Kosacoff, B. y Campanario, S. (2007). La revalorización de las materias primas y sus efectos en América Latina. Santiago de Chile: CEPAL.

Lilliston, B. y Ranallo, A. (2011). Excessive Speculation in Agriculture Commodities: Selected Writings from 2008-2011. Minneapolis, USA: IATP.

Losinno, B. y Conti, M. (junio, 2005). Evolución espacial y temporal de potasio en 30 años de agricultura continua en el noreste de la pampa ondulada. Informaciones Agronómicas de Hispanoamérica, (26). Recuperado de http://www.ipni.net/publication/ia-lacs.nsf/o/7B3892912AD15DF685257999 0060125F/\$FILE/Losinno-KPampa Ond\%2OIA\%202005.pdf

Luxemburg, R. (1964). The accumulation of Capital. New York, usa: Monthly Review Press.

Mandel, E. (1980). Las ondas largas del desarrollo capitalista: una interpretación marxista. Madrid, España: Siglo XXI.

Marx, K. (2006). El Capital. Crítica de la Economía Política. México DF, México: Siglo xxi Editores.

Montenegro, C., Gasparri, I., Manghi, E., Strada, M., Bono, J. y Parmuchi, M. G. (2004). Informe de avance sobre la deforestación en Argentina. Buenos Aires, Argentina: Dirección de Bosques, Secretaría de desarrollo sustentable.

Montenegro, J. (2008). Los límites del consenso. La propuesta del desarrollo territorial rural en América Latina. En B. Mançano Fernandes (Org.), Campesinato e agronegócio na América Latina: a questão agraria atual (pp. 249-274). San Pablo, Brasil: Expressão Popular.

Montenegro, J. (2011). Seis fragmentos para pensar hoy la continuidad de la modernidad/colonialidad en el proyecto autoreferenciado del desarrollo. En N. Giarracca (Comp.), Bicentenarios (otros), transiciones y resistencias (pp. 223-233). Buenos Aires, Argentina: Una Ventana.

Montenegro, R. A. (2006). Informe sobre los efectos de los plaguicidas en la salud y el ambiente. Necesidad de prohibir el uso de plaguicidas agropecuarios en áreas urbanas y periurbanas. Córdoba, Argentina: Ed. FUNAM. 
Morel, F. (s/f). Recomendaciones técnicas para productores de soja de la provincia de Misiones. Siembra. InTA. Recuperado de http://inta.gob.ar/documentos/recomendaciones-tecnicas-paraproductores-de-soja-de-la-provincia-de-misiones-siembra

Murillo, S. (2008). Colonizar el dolor. La interpelación ideológica del Banco Mundial en América Latina. El caso argentino desde Blumberg a Cromañón. Buenos Aires, Argentina: Clacso.

Neiman, G. (Dir.) (2010). Estudio sobre la demanda de trabajo en el agro argentino.Buenos Aires, Argentina: ciccus.

Oliva, A., Spira, A. and Multigner, L. (2001). Contribution of environmental factors to the risk of male infertility. Human Reproduction, 16(8), 1768-1776.

Ortiz, L., Santos, C., Pedace, R., Vélez Torres, I., Quiroa, S., Rojas, I. y Godinez, M. (2008). Fuelling destruction in Latin America the real price of the drive for agrofuels. Transgénicos Fora. Recuperado de http://stopogm.net/ sites/stopogm.net/files/RealPrice.pdf

Paganelli, A., Gnazzo, V., Acosta, H., López, S. y Carrasco, A. (2010). Glyphosate-Based Herbicides Produce Teratogenic Effects on Vertebrates by Impairing Retinoic Acid Signaling. Chemical Research in Toxicology, 23(10), 1586-1595.

Palmisano, T. (2015). Paradojas y resignificaciones del «cuidado del suelo» en el agronegocio argentino. La construcción de una consigna para el cambio tecnológico. Argumentos. Revista de crítica social, (15), 41-67.

Pengue, W. (2006). «Agua virtual». Agronegocio sojero y cuestiones económico ambientales futuras... Realidad Económica (223), 58-77.

Pierbattisti, D. (2008). La privatización de los cuerpos. La construcción de la proactividad neoliberal en el ámbito de las telecomunicaciones, 1991-2001. Buenos Aires, Argentina: Prometeo.

Portillo, J. y Conforti, A. (2009). Feedlotización de la ganadería argentina. Ponencia presentada en las vi Jornadas Interdisciplinarias de Estudios Agrarios y Agroindustriales. Buenos Aires, Argentina.

Schmidt, M. (2014). (Des)ordenamientos territoriales salteños. Una aproximación al contexto previo al Ordenamiento Territorial de Bosques Nativos en la provincia de Salta. Mundo Agrario, 15(28). Recuperado de http://www. mundoagrario.unlp.edu.ar/article/view/MAv15n28a12/5583

Schorr, M.; Manzanelli, P. y Basualdo, E. (2012). Élite empresarial y régimen económico en la Argentina: las grandes firmas en la posconvertibilidad. Buenos Aires, Argentina: Flacso.

Schumann, H. (2014). Especuladores del hambre. ¿Por qué suben los alimentos? Buenos Aires, Argentina: Mardulce.

Sili, M. (2016). Un modelo para comprender la dinámica de los territorios rurales. El caso de la Argentina. Mundo Agrario, 17(34). Recuperado de http://www.mundoagrario.unlp.edu.ar/article/view/ MAv17n34ao3/7223

Svampa, M. (2012). Consenso de los commodities, giro ecoterritorial y pensamiento crítico en América Latina. OSAL, 23(32), 15-38.

Svampa, M., Bottaro, L. y Sola Álvarez, M. (2009). La problemática de la minería a cielo abierto: Modelos de desarrollo, territorio y discursos dominantes. En M. Svampa (Coord.), Minería transnacional, narrativas del desarrollo y resistencias sociales (pp.29-50). Buenos Aires, Argentina: Biblos.

Teubal, M. y Palmisano, T. (2010). El conflicto agrario: características y proyecciones. En N. Giarracca y M. Teubal (Coord.), Del paro agrario a las elecciones de 2009. Tramas, reflexiones y debates (pp. 193-252). Buenos Aires: Antropofagia. 
Teubal, M. y Palmisano, T. (2013). Crisis alimentaria y crisis global: la Argentina de 2001/2002 y después. Realidad Económica, (279), 47-74.

Teubal, M. y Rodríguez, J. (2002). Agro y alimentos en la globalización. Una perspectiva crítica. Buenos Aires, Argentina: La colmena.

Teubal, M., Domínguez, D. y Sabatino, P. (2005). Transformaciones agrarias en Argentina. Agricultura industrial y sistema agroalimentario. En N. Giarracca y M. Teubal (Coord.), El campo argentino en la encrucijada. Estrategias y resistencias sociales, ecos en la ciudad (pp. 37-78). Buenos Aires, Argentina: Alianza Editorial.

Thwaites Rey, M. (2001). Alas rotas. La política de privatización y quiebra de Aerolíneas Argentinas. Buenos Aires, Argentina: Temas.

Tussie, D, (Comp.) (200o). Luces y sombras de una nueva relación. El Banco Interamericano de Desarrollo, el Banco Mundial y la Sociedad Civil. Buenos Aires, Argentina: FLAcso.

Valenzuela, C. (2005). Transformaciones y conflictos en el agro chaqueño durante los 90. Articulaciones territoriales de una nueva racionalidad productiva. Mundo agrario, 5(10). Recuperado de http:// www.mundoagrario.unlp.edu.ar/ article/view/v05n10ao2/1370

Ventimiglia, L., Carta, H. y Rillo, S. (septiembre, 200o). Exportación de nutrientes en campos agrícolas. Informaciones Agronómicas de Hispanoamérica, (7). Recuperado de http://www.ipni.net/publication/ia-lacs.nsf/o/A6AB235 F461783C48525799C0058ED55/\$FILE/ExpNut9deJulio.pdf 
